\title{
Hormones and mental health: where do we stand in the post-WHI era?
}

Ms. Jones is now 50 years old, and is facing a dilemma. Two weeks ago, her physician called, asking her to make an appointment to discuss her hormone treatment - "it is too risky”, she said. Ms. Jones left her doctor's office somewhat confused, and unsure about her health. After all, she had spent quite a few years struggling with symptoms of depression, difficulty in concentrating, fatigue, and problems sleeping due to intense hot flushes; several unsuccessful trials with different psychotropic medications had left Ms. Jones hopeless about her treatment. Around three months ago, she had initiated hormone replacement therapy (HRT) and had finally achieved some stability with combined use of antidepressants. Her quality of life had improved significantly. In addition, HRT was presented as an efficacious- and quite safe- strategy. Well, not anymore..

For many decades, women and health professionals were taught about the benefits and risks of hormone replacement therapy (HRT). Essentially, HRT had been administered to alleviate physical symptoms associated with the menopausal transition (short-term use of HRT), and to help in preventing the clinical consequences of an estrogen-deficient state, including osteoporosis and cardiovascular disease (long-term use of HRT). More recently, the list of benefits of short-term HRT was expanded, incorporating preliminary but promising findings on mood and cognition. Clinical trials demonstrated the efficacy of estradiol for the treatment of depressive disorders (including major depression) during perimenopause. ${ }^{1}$ In addition, estrogen therapy has been associated with an increase in $5 \mathrm{HT}(2 \mathrm{~A})$ receptor binding in prefrontal cortex of postmenopausal women, a potential pathway to explain its impact on mood and cognition. ${ }^{2}$ Results from large, prospective studies were highly desired, as they could provide more robust information on the risks and benefits of long-term HRT.

The results from the Women's Health Initiative Study (WHI) have caused both disappointment and apprehension. ${ }^{3}$ This multicenter, randomized, placebo-controlled primary prevention trial was designed to follow more than 16000 postmenopausal healthy women for eight years, and had to be closed early due to evidence of an increased risk for invasive breast cancer and cardiovascular events. The impact of HRT on "well-being" and sexual satisfaction was not significant. In addition, in a subgroup of subjects in which a higher risk for developing demen- tia had been identified (WHI Memory Study), HRT failed to prevent the development of cognitive deficits.

As a first reaction, physicians and patients felt betrayed, and many have decided to discontinue their HRT regimens. Still others who did not abandon their prescription hormones are now questioning their current treatment and potential alternatives. Most women and their doctors are now facing a difficult situation: how should they deal with menopause-related physical and emotional symptoms? Is there a role for HRT in the post-WHI era?

The results from the WHI study should be carefully interpreted. In fact, various Menopause Societies have expressed their concerns; investigators and seasoned clinicians have pointed out that some of these results were a "direct consequence of unsuitable population selection, terribly wide inclusion criteria, lack of adequate assessments, and the incongruous treatment choice". ${ }^{4}$

The benefits and safety of long-term use of conjugated estrogens and medroxiprogesterone (the most widely used hormone preparation) for postmenopausal women are now in question; research will undoubtedly increase in the search for safer alternatives, such as selective estrogen receptor modulators (SERMs) and diet supplementation, for prevention of menopause-related conditions.

On the other hand, clinicians and health professionals should continue considering many factors when advising women approaching menopause or postmenopausal on treatment choices:

- For those currently on HRT, an abrupt treatment discontinuation could lead to the occurrence or reemergence of somatic symptoms, interfering with sleep pattern, physical well-being and most probably mood. Thus, HRT discontinuation should be done gradually, and clinicians should be aware of an increased risk for mood instability, anxiety, and insomnia during or right after this process.

- The short-term use of HRT (up to three to five years) has not been considered unsafe, and still is the most efficacious treatment for vasomotor symptoms (i.e., night sweats, hot flushes). Importantly, recent data strongly suggest an increased risk for developing depression in perimenopausal women who report hot flushes, even in the absence of prior episodes of depression. Therefore, leaving this subpopulation symptomatic and untreated could widen this risk. 
- The results of the WHI study did not find evidence that the use of estrogen alone could lead to the same risks observed when used in combination with progestins. More studies are needed in this area.

- There are many HRT preparations available, including other types of estrogens and progestins (e.g., 17 b estradiol, micronized progesterone). The WHI study has yielded data only on the use of conjugated estrogens and medroxiprogesterone; long-term data on other HRT regimens are sparse, and certainly overdue. As already seen with various antidepressants, hormonal therapies differ with respect to absorption, metabolism, and bio-availability. It has been suggested, for example, that estradiol provides a more positive effect on mood and cognition, and could offer a different risk-benefit profile, given its similarity to endogenous sex hormones. Nonetheless, in the absence of more data, we cannot ensure the safety of switching patients to another hormone combination.

- Alternative treatments are not necessarily safe. Some of the so-called "natural" treatments for menopausal symptoms have a significant binding affinity for estrogen receptors, and may result in similar risks.

- Recent studies suggest that antidepressants (e.g. paroxetine, citalopram, venlafaxine) promote improvement of vasomotor symptoms, constituting an interesting alternative for menopausal women who experience depressive symptoms and vasomotor complaints. ${ }^{5}$

Ongoing studies suggest that hormone interventions (including testosterone, DHEA, etc) may play an important role in promoting well-being among aging men and women. More than ever, it is imperative to better delineate their clinical indications, and to learn more about risks and benefits associated with different hormonal preparations.

Claudio N Soares

Associate Director for Research

Center for Women's Mental Health

Massachusetts General Hospital, Harvard Medical School.

Boston

\section{References}

1. Soares CN, Almeida OP, Joffe H, Cohen LS. Efficacy of estradiol for the treatment of depressive disorders in perimenopausal women: a randomized, double-blind, placebo-controlled trial. Arch Gen Psychiatry 2001;58:529-34.

2. Kugaya A, Epperson CN, Zoghbi S, van Dyck CH, Hou Y, Fujita M et al. Increase in prefrontal cortex serotonin $2 \mathrm{~A}$ receptors following estrogen treatment in postmenopausal women. Am J Psychiatry 2003;160:1522-4.
3. Shumaker SA, Legault C, Thal L, Wallace RB, Ockene JK, Hendrix SL, WHIMS Investigators et al. Estrogen plus progestin and the incidence of dementia and mild cognitive impairment in postmenopausal women: the Women's Health Initiative Memory Study: a randomized controlled trial. JAMA 2003;289:2651-62.

4. Notelovitz M. The clinical practice impact of the Women's Health Initiative: political vs biologic correctness. Maturitas 2003;44:3-9.

5. Soares CN, Poitras JR, Shifren JL, Alexander AB, Cohen LS. Efficacy of citalopram as a monotherapy or as an adjunctive treatment for perimenopausal and postmenopausal women with depression and vasomotor symptoms. J Clin Psychiatry 2003;64:473-9. 